\title{
Identification of Suitable Plant Species for Vegetation Restoration at Manthali, Nepal
}

\author{
Sunita Poudel ${ }^{*}$, Mukesh Kumar Chettri ${ }^{2}$ \\ ${ }^{1}$ Department of Botany, Amrit Campus, Tribhuvan University, Kathmandu, Nepal \\ *E-mail: sunitapaudel628@gmail.com
}

(Received:26 December. 2021, Received in revised form: 20 April, Accepted: 4 May 2021, Available Online)

\section{Highlights}

- Vegetation analysis along the west-facing slope of the drought-prone mountain of Manthali was conducted.

- Based on high IVI value, 8 plant species of different life form were considered for the detailed study.

- Fourteen functional traits were considered for observation in those species.

- Annona squamosa and Abrus precatorius can be proposed as candidate species for early succession.

- This study identifies the suitable plant species for the restoration of degraded areas in a tropical zone.

\begin{abstract}
The present study aims to find out the suitable native plant species for restoring degraded land in the mountain from 450 $m$ asl to $819 \mathrm{~m}$ asl in Khurkot of Manthali. Altogether thirty quadrats were laid to observe frequency, density and coverage of plant species present there, and ultimately, IVI was calculated. Eight plant species - 3 trees like Acacia catechu, Aegle marmelos and Annona squamosa; 3 shrubs like Rhus parviflora, Cipadessa baccifera and Abrus precatorius (woody climber), and 2 herbs like Uraria lagopodiodes and Polypogon monospeliensis were considered for the detailed study based on their high IVI values. Altogether fourteen traits (growth form, life-span, phenology, Raunkiaer's life-form, canopy height, stem density, mean ramet distance, leaf size, specific leaf area, leaf thickness, seed mass, propagules mass, seed shape and nitrogen fixation) were studied in those eight species. Based on these traits, through this protocol, the plants were again categorized into five syndromes, i.e. establishment syndrome, dispersal syndrome, biomass producing syndrome, persistence syndrome and effect on environment syndrome. From this study, Annona squamosa and Abrus precatorius can be suggested as candidate species for early succession. Similarly, Aegle marmelos, Acacia catechu, Annona squamosa, Rhus parviflora and Abrus precatorius can be recommended to plant for restoration of degraded bushy lands, which are in the phase of secondary succession.
\end{abstract}

Keywords: succession, plant traits, trait syndromes, establishment, persistence

\section{Introduction}

Restoration can be defined as the process by which a disturbed site is restored to a pre-disturbance state, with similar environmental conditions, ecosystem structure and species complement [1]. Ecological restoration accelerates this process through a series of active interventions towards the ecological goal [2].

There is a certain process for restoration of the site with appropriate native species. Initially, one should choose appropriate plant species for plantation, then should prepare the site for restoration (by decreasing or removing invasive plant species, knowing about soil and making it suitable for plantation) and finally caring of the place [2].

For ecological restoration, it is better to use native plant species than exotic ones. A native (indigenous) species is one that occurs in a particular region, ecosystem, and habitat without direct or indirect human interactions [3]. Native species survived

${ }^{*}$ Corresponding author 
and repeatedly reproduced under the impulses of unstable climates, predation, disease, herbivory, competition, and all the other limitations and stresses imposed by an environment over multiple generations [4].

A total of sixteen traits were collected to closely mirror essential traits measured in other global studies [5]. Existing protocols were usually followed for their measurement [5,6] occasionally with slight modifications. The fourteen traits of native plants like growth form, life-span, phenology, Raunkiaer's life-form, canopy height, stem density, mean ramet distance, leaf size, specific leaf area, leaf thickness, seed mass, propagule mass, seed shape and nitrogen fixation were considered by Laurin [1].

Functional plant traits are well-defined characteristics [7] that relate to plant species' patterns of establishment, growth, and resource allocation and that evolved in response to abiotic environmental conditions and interactions with other species [8].

These plants traits can be divided into various plant trait syndrome, namely dispersal syndrome, establishment syndrome, biomass producing syndrome, persistent syndrome, effect on environment syndrome. The principal significance of this work is to find the best candidate species for the restoration of degraded land by dividing the dominant plant species according to its functional traits in the probable syndrome.

\section{Materials and Methods}

\section{Study area}

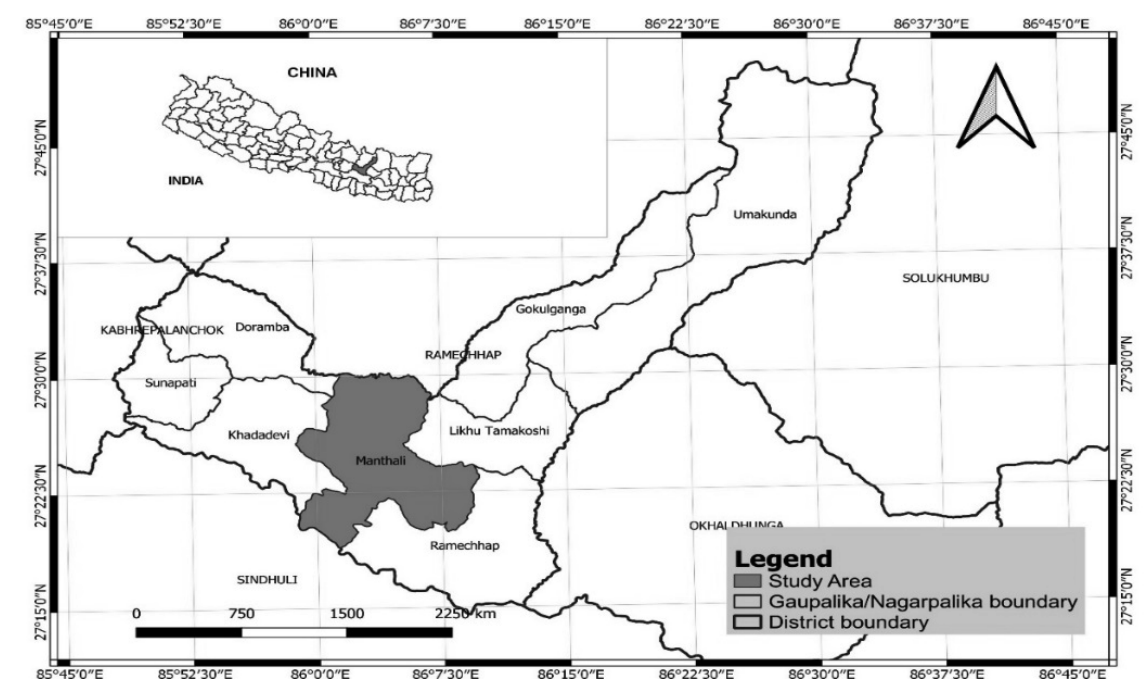

Fig 1: Map of the study area

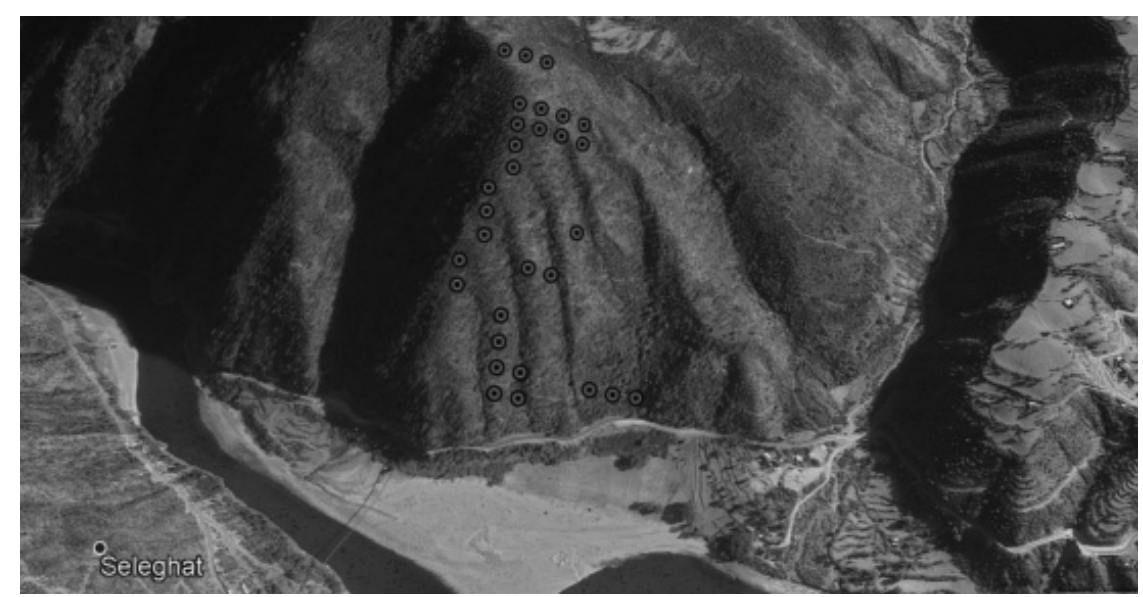

Fig 2: Study site with sampling plots

Source: www.google.com.np/maps/ 
Ramechhap has a diversified environment in the different ecological zone. The southern area of Ramechhap falls in the rain shadow zone of Mahabharat Hill, which extends up to Roshikhola catchment of Kavrepalanchowk [9]. Ramechhap was announced drought area by District Agriculture Coordination Committee on July 9, 2010.

\section{Soil}

Soil organic matter ranged from 1.2 to $2.4 \%$ indicating that some areas at higher altitudes have slightly degraded soil, but possibly it can help for the survival of the plants [10]. If organic matter is below $1 \%$, then it is not suitable for plantation [11]. Soil texture ranged from sandy to sandy loam. In the present study, soil pH ranged from 6.016 to 7.4 and is mostly within the optimal range, i.e. 6.5 to 7.5 as suggested by Lauchli and Grattan [12]. Hence, no adverse effect on the establishment of a plant in the study site is expected. Soil electrical conductivity of our study area ranged from 65.792 to $85.912 \mu \mathrm{S} / \mathrm{cm}$ [10], which is equivalent to 0.065 to $0.0859 \mathrm{dS} / \mathrm{m}$ and is within the range (less than $1 \mathrm{dS} / \mathrm{m}$ ). Hence, from these results, it can be concluded that soil properties like soil organic matter, soil $\mathrm{pH}$, soil electrical conductivity has no such adverse effect on the establishment of plant species.

\section{Sampling procedure}

Random sampling along the trail was conducted at Khurkot, Manthali, west-facing slope, ranging from $462 \mathrm{~m}$ to $820 \mathrm{~m}$ above sea level. Altogether 30 quadrats of $20 \times 20 \mathrm{~m}^{2}$ were used for tree species, 60 quadrats of $5 \times 5 \mathrm{~m}^{2}$ were used for shrub species and 60 quadrats of $2 \times 2 \mathrm{~m}^{2}$ were used for herb species. The frequency, density and coverage of all plant species present in the quadrat were recorded.

The analysis of the plant species distribution was conducted using the Importance Value Index (IVI) as the sum of relative density, relative frequency and relative coverage for comparison of the species dominance [13]. The IVI for a species was calculated as the sum of its relative frequency (R.F.), relative density (R.D.), and relative coverage (R.C.), using the following formula.

Importance Value Index (IVI) = R.D. + R.F. +R.C.

\section{Plant trait syndrome}

Various plant traits were noted, and candidate plants were selected for multiple trait syndromes like i) establishment syndrome, ii) dispersal syndrome, iii) biomass producing syndrome, iv) persistence syndrome, and v) effect on environment syndrome [1]. Based on different plant traits, the best candidate species were identified for various trait syndromes.

i. Establishment syndrome: For the establishment syndrome- large seed mass, higher SLA, short ramet distance, evergreen phenology and annual life span were the selection criteria.

ii. Dispersal syndrome: For the dispersal syndrome, plant traits like - less propagule mass, less seed mass, taller canopy height and round seed shape were the selection criteria.

iii. Biomass producing syndrome: For the biomass producing syndrome, plant traits like- less SLA, taller canopy height, high stem density, more leaf thickness, evergreen phenology were selection criteria.

iv. Persistence syndrome: Plant traits like taller canopy height, high stem density, less ramet distance, higher SLA, perennial life span, phanerophytes are selection criteria for persistence syndrome.

v. Effect on environment syndrome: Plants traits like tallest canopy height, large ramet distance, perennial life span, deciduous phenology and high leaf thickness were considered to select the candidate species for this syndrome.

\section{Plant traits}

Altogether 14 plant traits [5] were collected after the selection of species through sampling as described above.

\begin{tabular}{|l|l|}
\hline Traits & Methods \\
\hline Growth form & Plants were divided into herbs, shrubs, and trees. \\
\hline Life-span & Plants were divided into annual, biennial, perennial. \\
\hline Phenology & Plants were divided into evergreen and deciduous \\
\hline
\end{tabular}




\begin{tabular}{|l|l|}
\hline Raunkiaer's growth form & $\begin{array}{l}\text { All species were classified into the Raunkiaer's classification schemes, i.e. phanerophytes, } \\
\text { chamaephytes, hemicryptophytes, geophytes and therophytes }\end{array}$ \\
\hline Distance between ramets & The ramet distances were measured using a measuring tape. \\
\hline Canopy height & Canopy height measured using altimeter. \\
\hline Stem density & It is the dry weight of stem per volume [14]. \\
\hline SLA & $\begin{array}{l}\text { Specific leaf area (SLA) is the one-sided area of a fresh leaf, divided by its oven-dry mass } \\
{[15] .}\end{array}$ \\
\hline Leaf size & Leaf size was measured through Image J. \\
\hline Leaf thickness & Leaf thickness was calculated using micrometer screw gauge. \\
\hline Propagule mass & $\begin{array}{l}\text { Propagules were dried at } 80^{\circ} \mathrm{C} \text { for } 48 \text { hours and then were bulk weighed was taken from a } \\
\text { population. The number of propagules in that bulk weight was counted and then divided } \\
\text { the bulk weight by the number of propagules to determine the mean mass of a propagule. }\end{array}$ \\
\hline Seed mass & $\begin{array}{l}\text { They were dried at } 80^{\circ} \mathrm{C} \text { for } 48 \text { hours. They were weighed individually (15 seeds in total } \\
\text { for each species per site) or counted and bulk-weighed for smaller seeds (300 seeds) at } \\
0.1 \text { mg precision. }\end{array}$ \\
\hline Nitrogen fixation & $\begin{array}{l}\text { Leguminous plants were recorded as nitrogen-fixing plants, and non-leguminous plants } \\
\text { were searched for nitrogen fixation ability through Plants database [16]. }\end{array}$ \\
\hline Seed shape & Various shapes of seed were identified and related with seed banks in soil. \\
\hline
\end{tabular}

\section{Data analysis:}

To know if the plant species differ significantly or not for each plant trait, the data obtained ( $\mathrm{N}=30$ to 45$)$ were analyzed statistically using one way ANOVA followed by Duncan's multiple range test at $\mathrm{P}=0.05$ using SPSS version 27.0.1

\section{Results and Discussion}

Our result shows only 16 species from 450 masl to 819 masl. Based on IVI, eight plant species having high values were selected for further detailed study. Trees like Acacia catechu, Aegle marmelos and Annona squamosal, shrubs like Rhus parviflora, Cipadessa baccifera, Abrus precatorius (woody climber), and herbs like Uraria lagopodiodes and Polypogon monospeliensis were considered for the detailed study. Though the IVI value of Lantana camara and Ageratina adenophora were recorded very high but were not considered for detailed study because of their invasive nature [17].

\section{a. Plant trait syndromes}

\section{i. Establishment syndrome}

In the present study, among tree species, out of five traits (for establishment syndrome) three traits i.e.larger seed mass, highest SLA and evergreen/semi-deciduous, are fulfilled by Annona squamosa (Table 1). Hence, Annona squamosa can be considered the best establisher.

Table 1: Plant traits (seed mass, Specific Leaf Area, Ramet distance, Phenology, Life span) of establishment syndrome in dominant plants of Khurkot, Manthali

\begin{tabular}{|l|l|l|l|l|l|}
\hline Plants' name & Seed mass $(\mathbf{g})$ & SLA $\left(\mathbf{c m}^{2} / \mathbf{g}\right)$ & Ramet distance $(\mathbf{m})$ & Phenology & $\begin{array}{l}\text { Life } \\
\text { span }\end{array}$ \\
\hline Trees & $0.031 \pm 0.008 \mathrm{~A}$ & $91.94 \pm 3.65 \mathrm{~B}$ & $4.83 \pm 2.61 \mathrm{~A}$ & Deciduous & Perennial \\
\hline Acacia catechu & $0.058 \pm 0.003 \mathrm{~B}$ & $57.86 \pm 4.37 \mathrm{~A}$ & $5.06 \pm 2.89 \mathrm{~A}$ & Deciduous & Perennial \\
\hline Aegle marmelos & $0.183 \pm 0.001 \mathrm{C}$ & $129.29 \pm 7.07 \mathrm{C}$ & $3.76 \pm 0.92 \mathrm{~A}$ & $\begin{array}{l}\text { Evergreen/ } \\
\text { Semi-deciduous }\end{array}$ & Perennial \\
\hline Annona squamosa &
\end{tabular}




\begin{tabular}{|c|c|c|c|c|c|}
\hline \multicolumn{6}{|l|}{ Shrubs/ herbs } \\
\hline Rhus parviflora & $0.0560 \pm 0.002 \mathrm{~B}$ & $62.82 \pm 4.06 \mathrm{~A}$ & $2.96 \pm 1.66 \mathrm{~B}$ & Evergreen & Perennial \\
\hline Abrus precatorius & $0.151 \pm 0.047 \mathrm{C}$ & $184.77 \pm 2.41 \mathrm{D}$ & $2.86 \pm 0.70 \mathrm{~B}$ & Deciduous & Perennial \\
\hline Cipadessa baccifera & $0.030 \pm 0.022 \mathrm{AB}$ & $135.25 \pm 2.48 \mathrm{~B}$ & $3.93 \pm 1.45 \mathrm{~B}$ & Evergreen & Perennial \\
\hline Uraria lagopodiodes & $0.003 \pm 0.0009 \mathrm{~A}$ & $149.18 \pm 9.26 \mathrm{~B}, \mathrm{C}$ & $0.616 \pm 0.125 \mathrm{~A}$ & Deciduous & Perennial \\
\hline Polypogon monospeliensis & $0.0005 \pm 0.0008 \mathrm{~A}$ & $157.01 \pm 20.84 \mathrm{C}$ & $0.206 \pm 0.055 \mathrm{~A}$ & Deciduous & Annual \\
\hline
\end{tabular}

Same capital letters followed after mean \pm Standard deviation in a column do not differ significantly at $P=0.05$ according to Duncan's Multiple range tests followed after ANOVA.

The shrub Abrus had the highest seed mass and Specific Leaf Area value. Among herbs, Polypogon had the lowest ramet distance and annual life span (Table 1). Among shrubs and herbs, Abrus precatorius, Polypogon monspeliensis can be considered as good establisher.

\section{ii. Dispersal syndrome}

In the present study, among tree species out of four traits (for establishment syndrome), three characteristics, i.e. less propagule mass, less seed mass and large canopy height is fulfilled by Acacia catechu (Table 2). Hence, Acacia catechu can be considered to have the best dispersal syndrome.

The shrub Rhus parviflora had less propagule mass and round seed. Hence, it can be considered to have the best dispersal syndrome. Among the herbs, Uraria lagopodiodes and Polypogon monospeliensis had higher dispersal capacity as they had lowest propagule and seed mass. Among shrub and herbs; Rhus parviflora, Uraria lagopodiodes and Polypogon monospeliensis are the best dispersers (Table 2).

Table 2: Plant traits (propagule mass, seed mass, canopy height, seed shape) of dispersal syndrome in dominant plants of Khurkot, Manthali

\begin{tabular}{|c|c|c|c|c|}
\hline Plants' name & Propagule mass (g) & Seed mass (g) & Canopy height (m) & Seed shape \\
\hline \multicolumn{5}{|l|}{ Trees } \\
\hline Acacia catechu & $0.59 \pm 0.05 \mathrm{~A}$ & $0.031 \pm 0.008 \mathrm{~A}$ & $8.7 \pm 1.38 \mathrm{~B}$ & Ovate \\
\hline Aegle marmelos & $74.22 \pm 4.12 \mathrm{C}$ & $0.058 \pm 0.003 \mathrm{~B}$ & $7.64 \pm 1.39 \mathrm{~B}$ & Oblong \\
\hline Annona squamosa & $34.17 \pm 3.66 \mathrm{~B}$ & $0.183 \pm 0.001 \mathrm{C}$ & $5.03 \pm 0.74 \mathrm{~A}$ & Obovoid or elliptic \\
\hline \multicolumn{5}{|l|}{ Shrubs/Herbs } \\
\hline Rhus parviflora & $0.0049 \pm 0.0036 \mathrm{~A}$ & $0.056 \pm 0.0026 \mathrm{~B}$ & $5.47 \pm 0.68 \mathrm{C}$ & Round \\
\hline Abrus precatorius & $0.151 \pm 0.047 \mathrm{~B}$ & $0.151 \pm 0.047 \mathrm{C}$ & $8.55 \pm 1.24 \mathrm{D}$ & Oval \\
\hline Cipadessa baccifera & $0.21 \pm 0.017 \mathrm{C}$ & $0.0302 \pm 0.022 \mathrm{AB}$ & $1.75 \pm 0.47 \mathrm{~B}$ & Ovoid \\
\hline Uraria lagopodiodes & $0.0031 \pm 0.0009 \mathrm{~A}$ & $0.0031 \pm 0.0009 \mathrm{~A}$ & $0.06 \pm 0.01 \mathrm{~A}$ & Kidney-shaped \\
\hline Polypogon monospelieensis & $0.0005 \pm 0.00008 \mathrm{~A}$ & $0.0005 \pm 0.00008 \mathrm{~A}$ & $0.05 \pm 0.01 \mathrm{~A}$ & Oblong \\
\hline
\end{tabular}

Same capital letters followed after mean \pm Standard deviation in a column do not differ significantly at $\mathrm{P}=0.05$ according to Duncan's Mutiple range tests followed after ANOVA.

\section{ii. Biomass producing syndrome}

In the present study among tree species, out of five traits, two traits, i.e. lower SLA, higher leaf thickness, are fulfilled by 
Aegle marmelos (Table 3). Higher canopy height and higher stem density is fulfilled by Acacia catechu (Table 3). Hence, Aegle marmelos and Acacia catechu are considered to have the best biomass producing capacity.

Among shrubs and herbs, out of five traits, three traits, i.e. less Specific Leaf Area, evergreen phenology and higher leaf thickness, are fulfilled by Rhus parviflora (Table 3). Hence, Rhus parviflora will produce the best biomass among shrubs/herbs.

Table 3: Plant traits (Specific Leaf Area, canopy height, stem density, leaf thickness and phenology) of biomass producing syndrome in dominant plants of Khurkot, Manthali

\begin{tabular}{|c|c|c|c|c|c|}
\hline Plants' name & $\operatorname{SLA}\left(\mathrm{cm}^{2} / \mathrm{g}\right)$ & Canopy height (m) & Stem density $(\mathrm{g} / \mathrm{ml})$ & Leaf thickness (mm) & Phenology \\
\hline \multicolumn{6}{|l|}{ Trees } \\
\hline Acacia catechu & $91.94 \pm 3.65 \mathrm{~B}$ & $8.7 \pm 1.38 \mathrm{~B}$ & $0.87 \pm 0.05 \mathrm{C}$ & $0.01 \pm 0.00 \mathrm{~A}$ & Deciduous \\
\hline Aegle marmelos & $57.86 \pm 4.37 \mathrm{~A}$ & $7.64 \pm 1.39 \mathrm{~B}$ & $0.75 \pm 0.05 \mathrm{~B}$ & $0.56 \pm 0.10 \mathrm{~B}$ & Deciduous \\
\hline Annona squamosa & $129.29 \pm 7.07 \mathrm{C}$ & $5.03 \pm 0.74 \mathrm{~A}$ & $0.64 \pm 0.05 \mathrm{~A}$ & $0.43 \pm 0.13 \mathrm{~B}$ & Deciduous \\
\hline \multicolumn{6}{|l|}{ Shrubs/Herbs } \\
\hline Rhus parviflora & $62.82 \pm 4.06 \mathrm{~A}$ & $5.47 \pm 0.68 \mathrm{C}$ & $0.54 \pm 0.005 \mathrm{C}$ & $0.61 \pm 0.12 \mathrm{C}$ & sub-deciduous \\
\hline Abrus precatorius & $184.77 \pm 2.41 \mathrm{D}$ & $8.55 \pm 1.24 \mathrm{D}$ & $1.21 \pm 0.005 \mathrm{E}$ & $0.07 \pm 0.005 \mathrm{~A}$ & Deciduous \\
\hline $\begin{array}{l}\text { Cipadessa } \\
\text { baccifera }\end{array}$ & $135.25 \pm 2.48 \mathrm{~B}$ & $1.75 \pm 0.47 \mathrm{~B}$ & $0.62 \pm 0.003 \mathrm{D}$ & $0.0202 \pm 0.024 \mathrm{~B}$ & Evergreen \\
\hline $\begin{array}{l}\text { Uraria } \\
\text { lagopodiodes }\end{array}$ & $\begin{array}{l}149.18 \pm 9.26 \\
\text { B,C }\end{array}$ & $0.06 \pm 0.01 \mathrm{~A}$ & $0.02 \pm 0.005 \mathrm{~B}$ & $0.19 \pm 0.040 \mathrm{~B}$ & Deciduous \\
\hline $\begin{array}{l}\text { Polypogon } \\
\text { monospeliensis }\end{array}$ & $157.01 \pm 20.84 \mathrm{C}$ & $0.05 \pm 0.01 \mathrm{~A}$ & $0.01 \pm 0.00 \mathrm{~A}$ & $0.05 \pm 0.02 \mathrm{~A}$ & Deciduous \\
\hline
\end{tabular}

Same capital letters followed after mean \pm Standard deviation in a column do not differ significantly at $\mathrm{P}=0.05$ according to Duncan's Multiple range tests followed after ANOVA.

\section{iii. Persistence syndrome}

In the present study, among tree species, out of five traits, four traits, i.e. larger canopy height, higher stem density, phanerophytes growth form and perennial life span, is fulfilled by Acacia catechu. (Table 4). Annona squamosa also fulfills four traits among five, i.e. less ramet distance, higher SLA, perennial life span and phanerophytes (Table 4). So, Acacia catechu and Annona squamosa had the best persistence syndrome.

The shrub Abrus precatorius had a taller canopy height, higher stem density, higher SLA, perennial life span, phanerophytes growth form (Table 4). So, Abrus precatorius is the species having the best persistence syndrome.

Table 4: Plant traits (canopy height, stem density, ramet distance, Specific Leaf Area, Life span, Raunkiaer's growth form) of persistence syndrome in dominant plants of Khurkot, Manthali

\begin{tabular}{|c|c|c|c|c|c|c|}
\hline Plant's name & $\begin{array}{c}\text { Canopy } \\
\text { height }(m)\end{array}$ & $\begin{array}{c}\text { Stem density } \\
(\mathrm{g} / \mathrm{ml})\end{array}$ & $\begin{array}{c}\text { Ramet } \\
\text { distance(m) }\end{array}$ & $\operatorname{SLA}\left(\mathrm{cm}^{2} / \mathrm{g}\right)$ & Life span & $\begin{array}{l}\text { Raunkiaer's } \\
\text { growth form }\end{array}$ \\
\hline \multicolumn{7}{|l|}{ Trees } \\
\hline Acacia catechu & $8.7 \pm 1.38 \mathrm{~B}$ & $0.87 \pm 0.05 \mathrm{C}$ & $4.83 \pm 2.61 \mathrm{~A}$ & $91.94 \pm 3.65 \mathrm{~B}$ & Perennial & Phanerophytes \\
\hline Aegle marmelos & $7.64 \pm 1.39 \mathrm{~B}$ & $0.75 \pm 0.05 \mathrm{~B}$ & $5.06 \pm 2.89 \mathrm{~A}$ & $57.86 \pm 4.37 \mathrm{~A}$ & Perennial & Phanerophytes \\
\hline $\begin{array}{l}\text { Annona } \\
\text { squamosa }\end{array}$ & $5.03 \pm 0.74 \mathrm{~A}$ & $0.64 \pm 0.05 \mathrm{~A}$ & $3.76 \pm 0.92 \mathrm{~A}$ & $129.29 \pm 7.07 \mathrm{C}$ & Perennial & Phanerophytes \\
\hline
\end{tabular}




\begin{tabular}{|l|l|l|l|l|l|l|}
\hline \multicolumn{2}{|l|}{ Shrubs/Herbs } \\
\hline Rhus parviflora & $5.74 \pm 0.68 \mathrm{C}$ & $0.54 \pm 0.005 \mathrm{C}$ & $2.96 \pm 1.66 \mathrm{~B}$ & $62.82 \pm 4.06 \mathrm{~A}$ & Perennial & Phanerophytes \\
\hline $\begin{array}{l}\text { Abrus } \\
\text { precatorius }\end{array}$ & $8.55 \pm 1.24 \mathrm{D}$ & $1.21 \pm 0.005 \mathrm{E}$ & $4.60 \pm 1.10 \mathrm{~B}$ & $184.77 \pm 2.41 \mathrm{D}$ & Perennial & Phanerophytes \\
\hline $\begin{array}{l}\text { Cipadessa } \\
\text { baccifera }\end{array}$ & $1.75 \pm 0.47 \mathrm{~B}$ & $0.62 \pm 0.003 \mathrm{D}$ & $3.93 \pm 1.45 \mathrm{~B}$ & $135.25 \pm 2.48 \mathrm{~B}$ & Perennial & Phanerophytes \\
\hline $\begin{array}{l}\text { Uraria } \\
\text { lagopodiodes }\end{array}$ & $0.06 \pm 0.01 \mathrm{~A}$ & $0.02 \pm 0.005 \mathrm{~B}$ & $0.61 \pm 0.125 \mathrm{~A}$ & $\begin{array}{l}149.18 \pm 9.26 \\
\mathrm{BC}\end{array}$ & Annual & Chamaephytes \\
\hline $\begin{array}{l}\text { Polypogon } \\
\text { monospeliensis }\end{array}$ & $0.05 \pm 0.01 \mathrm{~A}$ & $0.01 \pm 0.00 \mathrm{~A}$ & $0.206 \pm 0.055 \mathrm{~A}$ & $157.01 \pm 20.84 \mathrm{C}$ & Annual & Chamaephytes \\
\hline
\end{tabular}

Same capital letters followed after mean \pm Standard deviation in a column do not differ significantly at $\mathrm{P}=0.05$ according to Duncan's Mutiple range tests followed after ANOVA.

\section{iv. Effect on environment syndrome}

In the present study, among tree species out of five traits, four traits, i.e. most extensive ramet distance, largest leaf thickness, perennial life span and deciduous phenology, are fulfilled by Aegle marmelos (Table 5). Hence, Aegle marmelos is the best candidate for the effect on the environment.

The shrub Abrus precatorius had the highest canopy height, perennial life span, deciduous phenology and nitrogen-fixing ability (Table 11). So among the shrubs, Abrus precatorius is the species having the best effect on environment syndrome.

Table 5: Plant traits (canopy height, ramet distance, leaf thickness, life span, and phenology) of effect on the environment syndrome in dominant plants of Khurkot, Manthali

\begin{tabular}{|c|c|c|c|c|c|c|}
\hline Plant's name & $\begin{array}{l}\text { Canopy } \\
\text { height (m) }\end{array}$ & $\begin{array}{l}\text { Ramet distance } \\
\text { (m) }\end{array}$ & $\begin{array}{l}\text { Leaf thickness } \\
(\mathrm{mm})\end{array}$ & Life span & Phenology & $\begin{array}{l}\text { Nitrogen- } \\
\text { fixing } \\
\text { capability }\end{array}$ \\
\hline \multicolumn{7}{|l|}{ Trees } \\
\hline Acacia catechu & $8.7 \pm 1.38 \mathrm{~B}$ & $4.83 \pm 2.61 \mathrm{~A}$ & $0.01 \pm 0.00 \mathrm{~A}$ & Perennial & Deciduous & Yes \\
\hline Aegle marmelos & $7.64 \pm 1.39 \mathrm{~B}$ & $5.06 \pm 2.89 \mathrm{~A}$ & $0.56 \pm 0.10 \mathrm{~B}$ & Perennial & Deciduous & Not known \\
\hline $\begin{array}{l}\text { Annona } \\
\text { squamosa }\end{array}$ & $5.03 \pm 0.74 \mathrm{~A}$ & $3.76 \pm 0.92 \mathrm{~A}$ & $0.43 \pm 0.13 \mathrm{~B}$ & Perennial & Deciduous & Not known \\
\hline \multicolumn{7}{|l|}{ Shrubs/herbs } \\
\hline Rhus parviflora & $5.47 \pm 0.68 \mathrm{C}$ & $2.96 \pm 1.66 \mathrm{~B}$ & $0.61 \pm 0.12 \mathrm{C}$ & Perennial & $\begin{array}{c}\text { Evergreen /Sub- } \\
\text { deciduous }\end{array}$ & Not known \\
\hline Abrus precatorius & $8.55 \pm 1.24 \mathrm{D}$ & $2.86 \pm 0.70 \mathrm{~B}$ & $0.07 \pm 0.005 \mathrm{~A}$ & Perennial & Deciduous & Yes \\
\hline $\begin{array}{l}\text { Cipadessa } \\
\text { baccifera }\end{array}$ & $1.75 \pm 0.47 \mathrm{~B}$ & $3.93 \pm 1.45 \mathrm{~B}$ & $0.0202 \pm 0.024 \mathrm{~B}$ & Perennial & Evergreen & Not known \\
\hline $\begin{array}{l}\text { Uraria } \\
\text { lagopodiodes }\end{array}$ & $0.06 \pm 0.01 \mathrm{~A}$ & $0.616 \pm 0.125 \mathrm{~A}$ & $0.19 \pm 0.040 \mathrm{~B}$ & Annual & Deciduous & Yes \\
\hline $\begin{array}{l}\text { Polypogon } \\
\text { monospeliensis }\end{array}$ & $0.05 \pm 0.01 \mathrm{~A}$ & $0.206 \pm 0.055 \mathrm{~A}$ & $0.05 \pm 0.02 \mathrm{~A}$ & Annual & Deciduous & Not known \\
\hline
\end{tabular}

Same capital letter followed after mean \pm Standard deviation in a column does not differ significantly at $\mathrm{P}=0.05$ according to Duncan's Multiple range tests followed after ANOVA. 


\section{b. Synopsis of all syndromes}

From all the above syndromes the name of plants which scored as a suitable candidate species are listed in Table 6 .

Table 6: Name of suitable candidate species of plants obtained from different syndrome for restoration

\begin{tabular}{|l|l|l|l|l|l|}
\hline $\begin{array}{l}\text { Growth } \\
\text { form }\end{array}$ & $\begin{array}{l}\text { Establishment } \\
\text { syndrome }\end{array}$ & Dispersal syndrome & $\begin{array}{l}\text { Biomass } \\
\text { producing } \\
\text { syndrome }\end{array}$ & $\begin{array}{l}\text { Persistence } \\
\text { syndrome }\end{array}$ & $\begin{array}{l}\text { Effect on } \\
\text { environment } \\
\text { syndrome }\end{array}$ \\
\hline Trees & Annona squamosa & Acacia catechu & $\begin{array}{l}\text { Aegle } \\
\text { marmelos/ } \\
\text { Acacia catechu }\end{array}$ & $\begin{array}{l}\text { Acacia catechu/ } \\
\text { Annona squamosa }\end{array}$ & Aegle marmelos \\
\hline Shrub /Herb & $\begin{array}{l}\text { Abrus precatorius/ } \\
\text { Polypogon } \\
\text { monospeliensis }\end{array}$ & $\begin{array}{l}\text { Rhus parviflora } \\
\text { Uraria lagopodiodes/ } \\
\text { Polypogon } \\
\text { monospeliensis }\end{array}$ & Rhus parviflora & Abrus precatorius & Abrus precatorius \\
\hline
\end{tabular}

\section{i. Early succession}

The present study suggests that Annona squamosa is suitable for early succession. Annona squamosa being very hardy, survives well under drought, salinity and marginal soil conditions. It grows very well, even on shallow soils at the mercy of nature [18]. The present study also suggested Abrus precatorius to be suitable for early succession, which had spread into new places very quickly, harmed other local plants in the area and became 'potentially invasive' very easily [19]. Abrus precatorius has an oceanic mode as the primary means of dispersal [20]. Abrus precatorius is already a key threat to the other plant communities in other parts of the world. For example, in certain places in Australia, it acts as a transformer species, changing the nature of the plant communities by smothering existing vegetation, both in the ground layer and canopy, altering the light climate and suppressing the regeneration of native species [19].

So, it might not be wise to use Abrus precatorius for early succession as it will harm any other species growing there. It might be wise to grow herbs like Polypogon monospeliensis that can be used for early succession.

\section{ii. For the land which already has few species}

The present study suggests Aegle mamelos, Acacia catechu, Annona squamosa, Rhus parviflora and Abrus precatorius as candidate species for the succession of land with few plant species. As we already discussed that it might not be wise to use Abrus precatorius for succession; it might be excluded.

Aegle marmelos has a reputation for thriving in a place where other plants cannot survive [21]. Aegle marmelos grows well in dry places [22], and it is distributed throughout the dry forest in hill and plain areas [23]. Annona squamosa (Sugar apple) grows well in hot, dry climates and adjusts in any kind of soil [24]. Sugar apple grows from sea level to 1,000 masl and requires no cold periods; and so, develops and grows well in relatively stable conditions of temperature [25]. Acacia catechu is one of the very few species which grows well in the degraded lands and could be very useful for the community plantations in Nepal [26].

\section{Conclusions}

On the basis of different syndrome studied, it can be concluded that the tree species like Annona squamosa is the best plant candidate for the establishment and also for persistence in the environment. Acacia catechu shows high dispersal as well as persistence syndrome. But the tree species Aegle marmelos is a good candidate for the high biomass producing syndrome, and hence its effect on environment is prevalent.

Among the shrubs, Abrus precatorius is a good establisher. It also persists and influences the environment. Rhus parviflora is candidate for dispersal and biomass production. The herb Polypogon monospeliensis is a good candidate for establishment.

In highly degraded areas, plantation of Annona squamosal among the trees, Abrus precatorius among shrubs and Polypogon monospeliensis among herbs should be encouraged to restore the bare land. For the restoration of less degraded areas i.e land with few plant species, tree species like Aegle marmelos, Acacia catechu and Annona squamosa, and among shrubs, Abrus precatorius and Rhus parviflora should be encouraged for plantation. 


\section{Acknowledgements}

The first author like to express sincere gratitude, deep sense of honour and appreciation to the supervisor, and to the Department of Botany, Amrit Campus, Tribhuvan University, Kathmandu for providing space and laboratory facilities.

\section{References}

1. C. Laurin. Identification of candidate plant species for restoration of newly created uplands in Subarctic: A functional ecology approach, M.Sc. Dissertation. School of Graduate Studies, Laurentian University, Sudbury, Ontario, 2012.

2. J. Dorner. An introduction to using native plants in restoration projects $1^{\text {st }}$ edition, Center for Urban Horticulture, University of Washington, and Publishers Environmental Protection Agency, 2002, pp.66.

3. R.T. Richards, C.C. Jeanne, R, Christopher. Use of native plants on federal lands: Policy and practice, Journal of Range Management, 1998, 51(6), 625-632.

4. R.W. Brown, R.S. Johnston, D.A. Johnson. Revegetation of disturbed alpine rangelands. In: Johnson, D. A., (eds.) Special management needs of alpine ecosystems. Range Science Series No. 5. Denver: Society for Range Management, 1979, pp. 1-16.

5. S. Díaz, J.G. Hodgson, K.Thompson, M. Cabido , J.H.C. Cornelissen, A. Jalili , G. Montserrat-Martí, J.P. Grime, F. Zarrinkamar., Y. Asri, S.R. Band, S. Basconcelo, P. Castro-Díez, G. Funes, B. Hamzehee, M. Khoshnevi, N. PérezHarguindeguy, M.C. Pérez-Rontomé, F.A. Shirvany, F. Vendramini, S. Yazdani, R.Abbas-Azimi, A. Bogaard, S. Boustani, M.Charles, L. Dehghan, L.de Torres-Espuny, V. Falczuk, J. Guerrero-Campo, A. Hynd, G. Jones, E. Kowsary, F. Kazemi-Saeed, M. Maestro-Martínez, A. Romo-Díez, S. Shaw , B. Siavash, P. Villar-Salvador, M.R. Zak. The plant traits that drive ecosystems: Evidence from three continents, Journal of Vegetation Science 2004, 15(3), 295-304.

6. J.H.C. Cornelissen, S. Lavorel, E. Garnier, S.Diaz., N. Buchmann, D. Gurvich, P. Reich, , H. Steege, H. Morgan, M. van der Heijden, J. Pausas, H. Poorter. A handbook of protocols for standardized and easy measurement of plant functional traits worldwide, Australian Journal of Botany, 2003, 51(4), 335-380. (DOI:10.1071/BT02124).

7. B.J. McGill, B.J. Enquist, E. Weiher, M. Westoby. Rebuilding community ecology from functional traits, Trends in Ecology and Evolution, 2006, 21(4), 178-185. (Doi.org/10.1016/j.tree.2006.02.002).

8. P.B. Reich, I.J. Wright, J. Cavender-Bares, J.M. Craine, J. Olek-syn, M. Westoby, M.B. Walters. The evolution of plant functional variation: traits, spectra, and strategies, International Journal of Plant Science, 2003, 164(S3), S143-S164. (Doi.org/10.1086/374368).

9. Shrestha, N.D. Acharya, N.B. Shrestha, H. Adhikari, S.K Shrestha. An assessment of drought in Ramechhap district. District Development Committee, Manthali, Ramechhap, 2015.

10. S. Poudel. Identification of suitable plant species for vegetation restoration at Manthali, M.Sc. Dissertation, Amrit Campus, Tribhuvan University, Nepal, 2020.

11. M. Brian. Key soil functional properties affected by soil organic matter - evidence from published literature. In: $I O P$ Conference Series Earth and Environmental Science. Australia, 2015, 1-5.

12. A. Lauchli, S. Grattan. Soil pH Extremes. In S. Shabala (eds.) Plant Stress Physiology. CAB Internationals, 2012, 194209. DOI:10.1079/9781845939953.0194.

13. G. Cottam, J. Curtis. The Use of Distance Measurements in Phytosociological Sampling, Ecology, 1956, 37(3), 451-460. (Doi.org/10.2307/1930167).

14. D.A. King, S.J. Davies, S. Tan, N.S. Noor. The role of wood density and stem support costs in the growth and mortality of tropical trees, Journal of Ecology, 2006, 94(3), 670-680. (Doi: 10.1111/j.1365-2745.2006.01112.x ). 
15. D. Vile, E. Garnier, B. Shipley, G. Laurent, M.L. Navas, C. Roumet, L. Lavorel, S. Diaz J.G. Hodgson, F. Lloret, G.F. Midgley, H. Poorter, M.C. Rutherford, P.J. Wilson, I.J. Wright. Specific Leaf Area and Dry Matter Content Estimate Thickness in Laminar Leaves, Annals of Botany, 2005, 96(6), 1129-1136. (Doi.org/10.1093/aob/mci264).

16. USDA and NRCS (United States Department of Agriculture and Natural Resources Conservation Service). The Plants - National Plant Data Team, Greensboro, North Carolina (2011). Retrieved from: https://plants.sc.egov.usda.gov. on: November 12, 2019.

17. S. Glass. Ecological Restoration as a Strategic Framework for Invasive Species Management Planning: The University of Wisconsin Experience 2004. Proceedings of 12th North American Prairie Conferences. pp 84.

18. P. Bhatnagar, J. Singh, M. Jain, B. Singh. Evaluation of land races of Custard apple (Annona squamosa L.), Plant Archives,2012, 12(2), 1045-1048.

19. K. Blood. Environmental weeds: A field guide for Southeast Australia. Cornell University. Publishers C.H. Jerrom and associates, Ithaca, New York, 2008, pp.112.

20. S.A. Ghazanfar, G. Keppel, S. Khan. Coastal vegetation of small islands near Viti Levu and Ovalau, Fiji, Journal of Botany, 2001, 39(4), 587-600.

21. K. Sampath Kumar, M. Umadevi, D. Bhowmik, D.S. Singh, A.S. Dutta. Recent trends in medicinal uses and health benefits of Indian Traditional Herbs Aegle marmelos, The Pharma Innovation, 2012, 1(4), 79-84.

22. D.K. Sekar, G. Kumar, L. Karthik, K.V.B. Rao, A review on pharmacological and phytochemical properties of Aegle marmelos (L.) Corr. Serr. (Rutaceae), Asian Journal of Plant Science and Research, 2011, 1(2), 8-17.

23. S. Vijayalakshmi, P. Venkatalakshmi. An updated review on the phytopharmacological significance of Aegle marmelos (L.), World Journal of Pharmaceutical Research, 2017, 6(16), 1390-1422.

24. A. Bhattacharya and R. Chakraverty, The pharmacological properties of Annona squamousa Linn: AReview, International journal of pharmacy and engineering, 2013, 4(2), 692-699.

25. M.F. Martínez., D. Miranda, S. Magnitskiy. Anatomy of sugar apple (Annona squamosa L.) seeds (Annonaceae), Agronomia Colombiana, 2013, 31(3), 279-287.

26. P. Jha, R.A. Mandal. Assessment of Growth Performance of Acacia catechu, International Journal of Advanced Research, 2019, 5(1), 1-5. (DOI:10.20431/2455-4316.0501005) 